Western University Scholarship@Western

Physics and Astronomy Publications

Physics and Astronomy Department

10-8-2009

\title{
The Lambert W Function and Quantum Statistics
}

Sree Ram Valluri

The University of Western Ontario, valluri@uwo.ca

M. Gil

The University of Western Ontario, 8mg48@queensu.ca

D.J. Jeffrey

The University of Western Ontario, djeffrey@uwo.ca

Shantanu Basu

The University of Western Ontario, basu@uwo.ca

Follow this and additional works at: https://ir.lib.uwo.ca/physicspub

Part of the Applied Mathematics Commons, Astrophysics and Astronomy Commons, and the Physics Commons

Citation of this paper:

Valluri, Sree Ram; Gil, M.; Jeffrey, D. J.; and Basu, Shantanu, "The Lambert W Function and Quantum Statistics" (2009). Physics and Astronomy Publications. 8.

https://ir.lib.uwo.ca/physicspub/8 


\title{
The Lambert $\boldsymbol{W}$ function and quantum statistics
}

\author{
S. R. Valluri, ${ }^{1,2, a)}$ M. Gil, ${ }^{1,2, b)}$ D. J. Jeffrey, ${ }^{2}$ and Shantanu Basu ${ }^{1}$ \\ ${ }^{1}$ Department of Physics and Astronomy, University of Western Ontario, Ontario N6A 3 K7, \\ Canada \\ ${ }^{2}$ Department of Applied Mathematics, University of Western Ontario, Ontario N6A 3K7, \\ Canada
}

(Received 3 June 2009; accepted 20 August 2009; published online 8 October 2009)

\begin{abstract}
We present some applications of the Lambert $W$ function ( $W$ function) to the formalism of quantum statistics (QS). We consider the problem of finding extrema in terms of energy for a general QS distribution, which involves the solution of a transcendental equation in terms of the $W$ function. We then present some applications of this formula including Bose-Einstein systems in $d$ dimensions, MaxwellBoltzmann systems, and black body radiation. We also show that for the appropriate parameter values, this formula reduces to an analytic expression in connection with Wien's displacement law that was found in a previous study. In addition, we show that for Maxwell-Boltzmann and Bose-Einstein systems, the $W$ function allows us to express the temperature of the system as a function of the thermodynamically relevant chemical potential, the particle density, and other parameters. Finally, we explore an indirect relationship of the $W$ function to the polylogarithm function and to the Lambert transform. (C) 2009 American Institute of Physics.
\end{abstract}

[doi:10.1063/1.3230482]

\section{INTRODUCTION}

It is always astonishing to see a mathematical function appear in seemingly unrelated applications. The multivalued transcendental $W$ function offers a very interesting example of this phenomenon. This function has undergone a renaissance in recent years with the advent of computer algebra systems such as MAPLE and MATHEMATICA. It has been called the Lambert $W$ function due to the fact that it is a logarithm of a special case of the Lambert series. ${ }^{1,2}$ There is a richness and diversity of multidisciplinary applications associated with this function.

Some of the earliest applications of the $W$ function relate to the counting of search trees and graphs, ${ }^{3}$ which occur in combinatorial applications in computer science as well as other disciplines including quantum statistics. One of the earliest applications of the $W$ function to physics was in the resolution of an anomaly in the calculation of exchange forces between two nuclei within the hydrogen molecular ion. ${ }^{4}$ In Ref. 5, the authors used the $W$ function to elucidate and develop some of the physics of Lagrangians for two-dimensional gravity. In relation to the work in Ref. 5, Ref. 6 presents a generalization of the $W$ function, which is used to provide exact solutions for general relativistic self-gravitating $N$-body systems in $(1+1)$ dimensions. Another application to physics is presented in Ref. 7. The authors used the $W$ function to derive Wien's displacement law in connection with the Planck black body spectral distribution and also applied it in the context of conformal mapping techniques to obtain the fringing fields of a parallel plate capacitor. In Refs. 8 and 9 the $W$ function is applied to problems in statistical mechanics. Germani and Liguori ${ }^{10}$ incorporated the $W$ function in their study of the slingshot primordial power spectrum in the

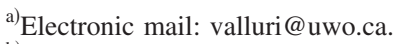

${ }^{b)}$ Present address: Department of Mathematics and Statistics, Queen's University.
} 
cosmological slingshot scenario. They computed the cosmic microwave background temperature and polarization power spectra that are in excellent match with the results of the Wilkinson microwave anisotropy probe 3-year best fit results.

As stated above, the diversity of applications of the $W$ function are widespread. In electromagnetics, numerical algorithms of this function have been incorporated into popular mathematical computation packages such as MAPLE and MATHEMATICA, allowing for efficient and rapid solutions and eliminating the need for graphical problem solving techniques. ${ }^{11}$ The $W$ function has been applied to semiconductor physics, where it has been used to find a solution to the problem of inverting the gate voltage versus channel surface potential equation. ${ }^{12}$ It has also been used in laser and passive electro-optic systems to obtain a solution of the ladar-lidar equation. ${ }^{13,14}$ In solar physics, new views of solar wind have been found with the $W$ function, allowing the analytical derivation of both the outflow speed and the mass loss rate of the solar wind for certain illustrative approximations. ${ }^{15}$

In the present work, we present yet another application of the $W$ function to mathematical physics in the context of quantum statistics. We consider the problem of finding analytic formulas for the critical points in energy of several relevant physical quantities and present some applications to Bose-Einstein and classical Maxwell-Boltzmann statistics. We note that this analysis is also relevant for the case of quantum fractional statistics, such as anyon and exclusion statistics. ${ }^{16}$ Furthermore, we use the $W$ function to develop inversion formulas for the temperature of the system as a function of the chemical potential for Bose-Einstein and Maxwell-Boltzmann statistics. The case to Fermi-Dirac statistics has also been considered but will be presented in connection to another work. The same analysis is also relevant for other cases of specialized twodimensional and four-dimensional Bose-Einstein systems. ${ }^{17}$ In Sec. II of this paper, we introduce the fundamental quantum statistical equations and define the relevant physical quantities pertaining to our analysis. This is followed by a brief introduction on the $W$ function in Sec. III. Section IV develops the analytic formula for the energy critical points. In Sec. V, we apply this analysis to particular distributions. In Sec. VI we present the inversion of temperature as a function of the chemical potential for Bose-Einstein and Maxwell-Boltzmann statistics. Section VII explores an indirect relationship of the $W$ function to the Lambert transform and the polylogarithm. Finally, Sec. VIII presents the conclusions of this work.

\section{QUANTUM STATISTICS}

We provide an overview of the statistics that describe a system of noninteracting particles confined, for example, within a cube of volume $V$ with impenetrable walls at finite temperature $T$. When we consider a system composed by a large number of noninteracting equivalent particles, we have the following distribution function: ${ }^{18}$

$$
\langle n(E)\rangle=[\exp (\beta E+\alpha)+\gamma]^{-1}, \quad \beta=\frac{1}{k T},
$$

where $E$ is assumed to be a continuous variable, $T$ is the temperature, $k$ is the Boltzmann constant, and $\alpha=-\beta \mu$, with $\beta$ defined as above and $\mu$ is the chemical potential. The term $e^{-\alpha} \equiv z$ in (1) is the fugacity of the system. In the case of classical particles, i.e., Maxwell-Boltzmann statistics, we have $\gamma=0$, and the particles are distinguishable. For Bose-Einstein and Fermi-Dirac systems, $\gamma$ takes on the values -1 and 1 , respectively. The particles are taken to be indistinguishable when their spin is of the form $s=n / 2, n \in\{0,1,2,3, \ldots\}$. For bosons, $s=0,+1,+2, \ldots$, we have $\gamma=-1$, whereas for fermions, $s=(2 p+1) / 2 p \in\{0,1,2, \ldots\}$, we have $\gamma=1$. In our analysis, we allow $\gamma$ to take any rational number in $[-1,1]$ to allow for the possibility of fractional statistics.

We can express the number of particles per unit volume within the energy interval $(E, E+d E)$, the number density $\rho$, and the total internal energy $U$ per unit volume as follows: ${ }^{18}$

$$
n(E) \frac{d E}{V}=\frac{(2 s+1)}{4 \pi^{2}}\left(\frac{2 m}{\hbar^{2}}\right)^{3 / 2} \frac{E^{1 / 2}}{\exp (\beta E+\alpha)+\gamma} d E,
$$




$$
\begin{gathered}
\rho=\frac{1}{V} \int_{0}^{\infty} n(E) d E=\frac{(2 s+1)}{4 \pi^{2}}\left(\frac{2 m}{\hbar^{2}}\right)^{3 / 2} \int_{0}^{\infty} \frac{E^{1 / 2} d E}{\exp (\beta E+\alpha)+\gamma}, \\
U=\frac{1}{V} \int_{0}^{\infty} E n(E) d E=\frac{(2 s+1)}{4 \pi^{2}}\left(\frac{2 m}{\hbar^{2}}\right)^{3 / 2} \int_{0}^{\infty} \frac{E^{3 / 2} d E}{\exp (\beta E+\alpha)+\gamma},
\end{gathered}
$$

with $m$ being the mass of the particles and $\hbar=h / 2 \pi$ where $h$ is Planck's constant.

In a series of remarkable papers, Lee ${ }^{19,20}$ showed a variety of applications of the polylogarithm function to Fermi-Dirac, Bose-Einstein, and classical Maxwell-Boltzmann statistics, which include a convenient description of physical quantities such as the density, energy, and chemical potential in terms of this function. For the case of Bose-Einstein statistics, $\gamma=-1$, the integrals in the previous equations can be evaluated in the following way. Consider the integral

$$
I_{r}=\int_{0}^{\infty} \frac{E^{r}}{e^{\beta E+\alpha}-1} d E \quad r \in \mathbb{R}^{+} .
$$

Using the transformation $x=\beta E=E /(k T)$ and multiplying both numerator and denominator by $\exp [-(x+\alpha)]$ we can bring it to the following form:

$$
\beta^{-(r+1)} \int_{0}^{\infty} x^{r} e^{-(x+\alpha)} \frac{1}{1-e^{-(x+\alpha)}} d x .
$$

For Bose-Einstein systems, the chemical potential $\mu$ is negative, ${ }^{21}$ so that $x+\alpha>0$ for all points over the region of integration, and we can expand the integrand as a geometric series. Thus we obtain the following result:

$$
I_{r}=\beta^{-(r+1)} \Gamma(r+1) \operatorname{Li}_{r+1}\left(e^{-\alpha}\right),
$$

where

$$
\Gamma(x)=\int_{0}^{\infty} t^{x-1} e^{-t} d t
$$

is the Gamma function and

$$
\operatorname{Li}_{r}(x)=\sum_{k=1}^{\infty} \frac{x^{k}}{k^{r}}
$$

is the polylogarithm function. ${ }^{22}$ From the definition of the polylogarithm, we see that it is related to the Riemann zeta function by the identity $\zeta(z)=\operatorname{Li}_{z}(1)$.

The case for $\gamma=1$ must be treated differently since $\mu$ can in this case be positive, which prevents us from simply using the geometric series expansion. However, the closed form solution turns out to be extremely similar to the one for the Bose-Einstein case and is given by ${ }^{23}$

$$
I_{r}=-\beta^{-(r+1)} \Gamma(r+1) \operatorname{Li}_{r+1}\left(-e^{-\alpha}\right) .
$$

Although not immediately relevant for our analysis, we include the corresponding expression when $\gamma=0$ for completeness:

$$
I_{r}=e^{-\alpha} \beta^{-(r+1)} \Gamma(r+1) .
$$

From the above results we find, for example, that the density $\rho$ introduced in Eq. (3) is given by 


$$
\begin{aligned}
\rho & =\frac{(2 s+1)}{4 \pi^{2}}\left(\frac{2 m}{\hbar^{2}}\right)^{3 / 2} \beta^{-3 / 2} \Gamma(3 / 2) \begin{cases}\operatorname{Li}_{3 / 2}\left(e^{-\alpha}\right), & \gamma=-1 \\
-\mathrm{Li}_{3 / 2}\left(-e^{-\alpha}\right), & \gamma=1\end{cases} \\
& =\frac{(2 s+1)}{4 \pi^{2}}\left(\frac{2 m}{\hbar^{2}}\right)^{3 / 2} \beta^{-3 / 2} \frac{\sqrt{\pi}}{2} \begin{cases}\sum_{k=1}^{\infty} \frac{\left(e^{\beta \mu}\right)^{k}}{k^{3 / 2}}, & \gamma=-1, \\
\infty & \left(-e^{\beta \mu}\right)^{k} \\
-\sum_{k=1}^{\infty} \frac{\left(k^{3 / 2}\right.}{} & \gamma=1 .\end{cases}
\end{aligned}
$$

This result will be used in Sec. VI. In the following section we give a brief introduction to the $W$ function and some of the mathematical properties that become relevant for this analysis. For a very thorough treatment of the history, properties, and applications of the $W$ function, see Ref. 24.

\section{THE LAMBERT W FUNCTION}

The $W$ function is defined as the multivalued function which solves the following equation:

$$
W(z) \exp (W(z))=z, \quad z \in \mathbb{C},
$$

or, equivalently, as the multivalued inverse of the function $f: z \rightarrow z e^{z}$. Equation (12) always has an infinite number of solutions, hence the multivaluedness of the $W$ function. These solutions are indexed by the integer variable $j$. Thus we say that (12) is solved by the branches of the $W$ function, $W_{j}$, for $j \in \mathbb{Z}$. Of special relevance to physics and engineering applications are the solutions of (12) when the argument is purely real. In this case there can be at most two real solutions, corresponding to the branches $W_{0}$ and $W_{-1}$, where $W_{0}$ is the principal branch of the $W$ function. For real solutions to exist, we must require that $z \in(-1 / e, \infty)$, in which case $W_{0}(z) \in$ $[-1, \infty)$ and $W_{-1}(z) \in(-\infty,-1]$. Moreover, $W_{0}(z)<0$ if $z \in(-1 / e, 0)$ and $W_{0}(z) \geq 0$ for $z \in[0, \infty)$. The branches $W_{0}$ and $W_{-1}$ are monotonically increasing and monotonically decreasing, respectively. All other branches of $W$ are always complex.

Besides the theoretical advantages of providing an adequate analytical formalism for a given problem, another advantage of solving problems in terms of the $W$ function is the availability of libraries in computer algebra systems, which allows for a convenient way to obtain values, expansions, plots, etc., of the quantity being solved for.

As a final remark we note that although, to our knowledge, the $W$ function is not connected to the Lambert transform of a function in a general sense, the results from this work show there exists a family of functions where an indirect relationship does indeed hold, which is shown in Sec. VII.

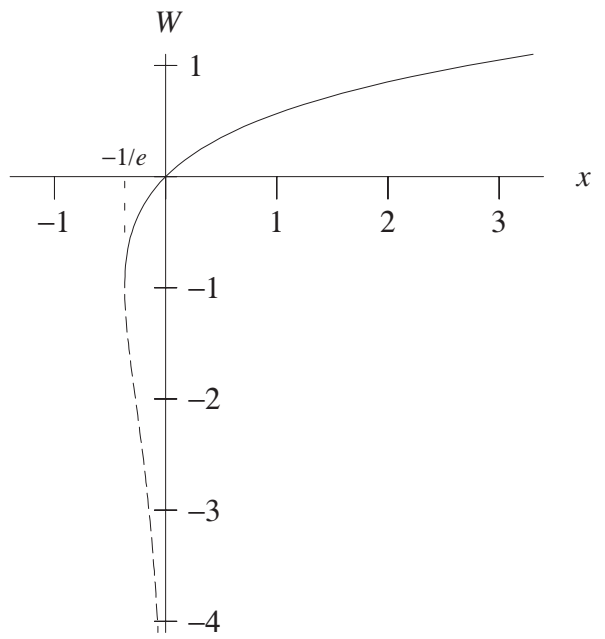

FIG. 1. The two real branches of the $W$ function. The solid line represents $W_{0}$ and the dashed line represents $W_{-1}$. 

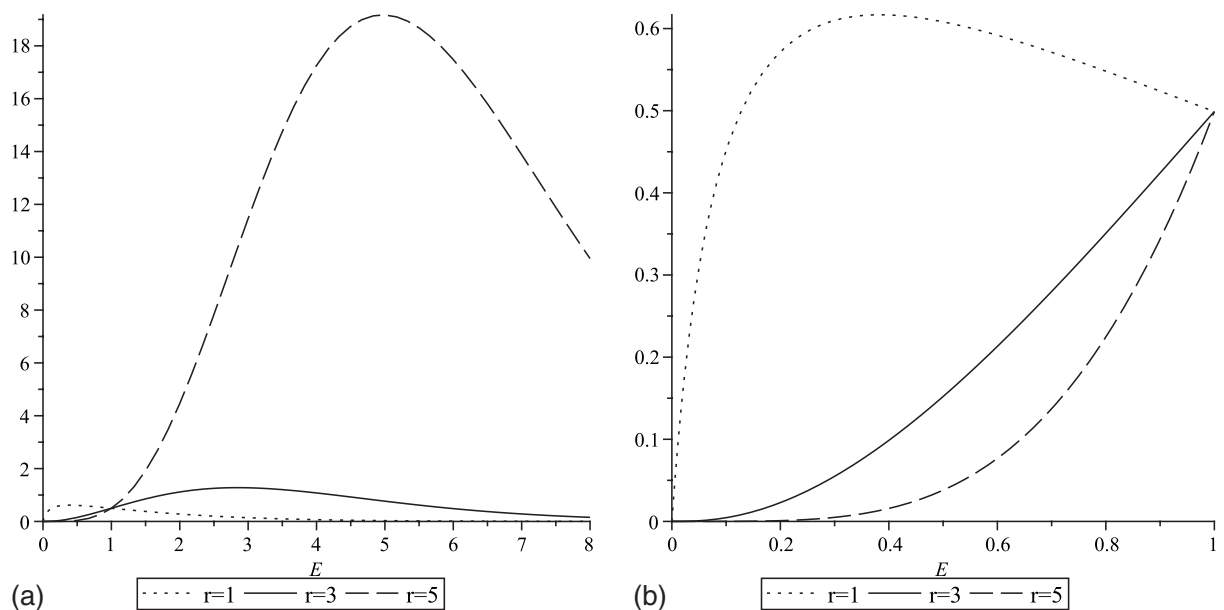

FIG. 2. Plots of $g_{r}$ for $\mu=1 / 10$ in units where $k T=1$.

\section{ENERGY EXTREMA OF QUANTUM STATISTICAL DISTRIBUTIONS}

Following a similar approach as in Ref. 18 we can express (3) and (4) as

$$
\begin{gathered}
\rho=\rho_{0}+\frac{(2 s+1)}{4 \pi^{2}}\left(\frac{2 m}{\hbar^{2}}\right)^{3 / 2} \int_{0}^{\infty} g_{1 / 2}(E) d E, \\
U=\frac{(2 s+1)}{4 \pi^{2}}\left(\frac{2 m}{\hbar^{2}}\right)^{3 / 2} \int_{0}^{\infty} g_{3 / 2}(E) d E,
\end{gathered}
$$

respectively, where

$$
g_{r}(E)=\frac{E^{r}}{e^{\beta E+\alpha}+\gamma}, \quad \beta=\frac{1}{k T},
$$

and $\rho_{0}$ is the density of particles in the ground state with $E=0$ for Bose-Einstein particles, and is taken to be 0 for Fermi-Dirac particles. ${ }^{18}$ We note that in Ref. 18 the actual definitions are slightly different.

Now, somewhat more generally, suppose we are interested in some arbitrary physical quantity $M$, which we can expressed as

$$
M=A \int_{0}^{\infty} h(E) d E,
$$

where

$$
h(E)=\frac{E^{f(r)}}{e^{\beta E+\alpha}+\gamma}
$$

for some real-valued continuous function $f: \mathbb{R} \rightarrow \mathbb{R}^{+}$and $A \in \mathbb{R}$. We interpret the quantities $M$ as generalized moments of the energy. In the case of the density and the energy in the previous equations, we have $h(E)=g_{1 / 2}(E)$ and $g_{3 / 2}(E)$, respectively, which we can think of as the $1 / 2$ and the $3 / 2$ energy moments. Then it will be of primary interest to perform an analysis on the distributions $h(E)$. A natural question that would arise is for what values of the parameters will such distributions exhibit a peak. However, if we want to find extrema in $E$ we are faced with a transcendental equation. Although it is not possible to formulate a solution in terms of elementary 
functions, we show that it is in fact possible to find an analytical formula for the values of the critical points in $E$ over a wide range of the parameters $h(E), \alpha$, and $\gamma$, by using the $W$ function. In fact, this approach has been used in Ref. 7 to find an expression for the energy extremum of the Planck spectral black body distribution as a function of the wavelength and temperature. In Sec. V B we show that the general expression derived in this paper reduces to the result found in Ref. 7 under the relevant parameter values.

Taking $E$ to be a continuous variable and assuming we fix the parameters $\alpha, r, \gamma$, and temperature $T$, we proceed to finding extrema in $E$ interior on $(0, \infty)$ : From (16) we find

$$
\frac{\partial h}{\partial E}=\frac{1}{\left[e^{\beta E+\alpha}+\gamma\right]^{2}}\left[f(r) E^{f(r)-1}\left(e^{\beta E+\alpha}+\gamma\right)-\beta E^{f(r)} e^{\beta E+\alpha}\right],
$$

where $\alpha=-\beta \mu=-\mu / k T$. It is worth noting that here that we are considering cases where we treat $\alpha$ as an exogenous variable, not directly dependent on $E$. Also (17) is always defined, since the only potential complication would arise in $E^{f(r)}$ for $E=0$, but this point is not considered in the analysis. Setting $\partial h / \partial E=0$ and rearranging, we obtain the following:

$$
[\beta E-f(r)] e^{\beta E-f(r)}=f(r) \gamma e^{-[\alpha+f(r)]} .
$$

Now we apply the $W$ function to both sides of this equation. Thus we have

$$
E_{c}=k T\left[f(r)+W_{j}\left(f(r) \gamma e^{-[\alpha+f(r)]}\right)\right],
$$

where $E_{c}$ denotes the critical point in temperature, and we have substituted $1 / \beta=k T$. We observe that in principle $j \in \mathbb{Z}$, though we focus on the cases $j \in\{0,-1\}$ where both the arguments and the range of $W_{j}$ in Eq. (19) are real. Hence, given the domain of $W_{0}$ and $W_{-1}$ we must satisfy

$$
f(r) \gamma e^{-[\alpha+f(r)]} \geq-\frac{1}{e} .
$$

Since $f(r) \geq 0$, it is clear that this will be satisfied for any statistics having $\gamma \geq 0$. In particular, this ensures that the expression for the critical point always exists for Fermi-Dirac and MaxwellBoltzmann statistics. Also, when $\gamma \geq 0$, we will always have the solution in terms of $W_{0}(z)$.

The $W$ function defines the omega Wright function via the following identity: ${ }^{25}$

$$
\omega(z)=W_{\mathcal{K}(z)}\left(e^{z}\right),
$$

where $\mathcal{K}(z)=\lfloor(\pi-\mathfrak{I}(z)) / 2 \pi\rfloor$, and $\lfloor\cdots\rfloor$ denotes the floor function. Thus, for $x \in \mathbb{R}$ we have

$$
\omega(x)=W_{0}\left(e^{x}\right) .
$$

Under the assumption that $\gamma f(r)>0$, we may apply (22) to (19) to obtain

$$
E_{c}=k T(f(r)+\omega[\log (f(r) \gamma)-(\alpha+f(r))]) .
$$

For the case $\gamma \in[-1,0)$ it may be useful to express (20) as a constraint on the fugacity $z=e^{-\alpha}$. Thus,

$$
-f(r)|\gamma| e^{-[\alpha+f(r)]} \geq-\frac{1}{e} \Rightarrow z=e^{-\alpha} \leq \frac{1}{|\gamma|} \frac{e^{f(r)-1}}{f(r)} .
$$

We also note that the two potential solutions, associated with the branches $W_{0}$ and $W_{-1}$, may both in fact correspond to physically relevant situations. This fact arises as a result of two things. First of all, taking $T>0$, the fact that $E>0$ requires us to have 


$$
W_{j}\left(f(r) \gamma e^{-[\alpha+f(r)]}\right)>-f(r) .
$$

Taking $h(E)=g_{r}(E)$ as defined in (15), so that $f(r)=r \geq 1$, then $-f(r) \leq-1 \leq W_{0}(z) \quad \forall z$ $\in[-1 / e, \infty)$; hence $W_{0}(z)$ automatically provides a physically relevant solution whenever the argument lies in the domain of $W(z)$ as discussed above. Moreover, $\forall z \in[-1 / e, 0), W_{-1}(z) \in($ $-\infty,-1)$, so that as $r$ increases, we will have an increasing region of the domain where $W_{-1}(z)$ will satisfy (25). Once $r$ is specified it will be necessary to determine whether the region in question corresponds to physically relevant conditions, as $W_{-1}(z)$ is only defined over $z \in(-1 / e, 0)$. In particular, it is necessary but not sufficient that $\gamma<0$, as, for example, in Bose-Einstein statistics.

A second source for the possibility of the two-branch solution relates to the idea of negative temperature. Although traditionally it is always assumed that $T>0$, negative temperature has a physically sensible interpretation. A system with negative temperature is not cold. It is in fact very hot, transferring energy to any positive-temperature system in contact with it. ${ }^{21,26}$ Negative temperatures can be considered for a closed thermodynamic system in thermodynamic equilibrium. Such systems have an energetic upper limit to their allowed states. This concept is useful in nuclear spin resonance experiments where isolation of the spin system can be attained. ${ }^{27}$ If we allow for such a situation in our analysis, then keeping $E>0$ requires that we satisfy

$$
W_{j}\left(f(r) \gamma e^{-[\alpha+f(r)]}\right)<-f(r) .
$$

In this case, and still assuming $h(E)=g_{r}(E)$ with $r \geq 1, W_{0}(z)$ no longer provides a solution at all [except possibly for the branch point $z=-1 / e$ where $W_{0}(-1 / e)=W_{-1}(-1 / e)=-1$ ] due to $-r \leq-1$. However, for any $r>1$, there is always a subset of $(-1 / e, 0)$ where $W_{-1}$ does satisfy $(26)$, the physical significance of which would have to be assessed in each particular case of $r$. As was mentioned above this requires $\gamma<0$ given the domain of $W_{-1}(z)$. In principle, for $\mu$ and or $T$ complex, there is the interesting possibility of other branches $W_{j}(z), j \neq 0,-1$ providing a solution in Eq. (19).

\section{APPLICATION TO SPECIFIC QUANTUM STATISTICAL DISTRIBUTIONS}

Section IV presented an analytic solution for the extremum in energy for the general distribution

$$
h(E)=\frac{E^{f(r)}}{e^{\beta E+\alpha}+\gamma} .
$$

In this section we consider application of (19) to special cases of such distribution. In particular, we show that under the appropriate parameters, our solution (19) reduces to the solution given in Ref. 7 for the analytic derivation of Wien's constant.

\section{A. Maxwell-Boltzmann distribution}

For Maxwell-Boltzmann systems we have $\gamma=0 .{ }^{18}$ As was pointed out above, $\gamma=0$ satisfies the condition for convergence (20), and $g_{3 / 2}$ corresponds to the distribution for the total internal energy of the system. Thus, solution (19) becomes

$$
E_{c}=k T\left[\frac{3}{2}+W_{0}(0)\right]=\frac{3}{2} k T,
$$

which is consistent with the equipartition theorem in statistical mechanics.

\section{B. Wien's displacement law}

In Ref. 7, the authors considered the Planck spectral distribution given by 


$$
\rho(\lambda, T)=\frac{8 \pi h c / \lambda^{5}}{\exp (h c / \lambda k T)-1},
$$

where $\lambda$ is the wavelength, $T$ is the temperature, $c$ is the speed of light, and $k$ is the Boltzmann constant. Since this corresponds to a system of photons, we have $E=h c / \lambda$ so that (28) can be written as

$$
\rho(\lambda, T)=\frac{8 \pi}{(h c)^{4}} g_{5}(E, \gamma=-1, \alpha=0),
$$

and the solution is given by (19) as

$$
E_{c}=k T\left[5+W_{0}\left(-5 e^{-5}\right)\right] .
$$

Note that we are using $W_{0}$ since $W_{-1}$ results in $E=0$. Then

$$
\lambda_{\max } T=\frac{h c / k}{5+W_{0}\left(-5 e^{-5}\right)}=2.893 \times 10^{-3} \mathrm{~m} \mathrm{~K},
$$

which is precisely what was found in Ref. 7.

\section{Bose-Einstein condensates}

For the Bose-Einstein distribution we have $\gamma=-1$, and $h(E)=g_{r}(E)$, so that $f(r)=r \geq 1$. As we noted above in (24), negative values for $\gamma$ place constraints on physically relevant quantities such as the fugacity $e^{-\alpha}$. In terms of $\gamma$ alone, the strongest constraint on the fugacity will then come for $|\gamma|=1$, given that this is the minimum of the right hand side of (24). A direct application of Eq. (19) for the case of Bose-Einstein distributions can be found in Ref. 28. The authors considered the particle density distribution in relative momentum space and were interested in finding the maximum of the expression

$$
f_{3}(x, \bar{\mu})=\frac{x^{2}}{e^{x^{2}+\bar{\mu}}-1},
$$

where $x$ is the relative momentum $x=p / p_{T}, p_{T}=\sqrt{2 m T}$, and $\bar{\mu}=-\mu / T$. By letting $x^{2}=\beta E$ we find

$$
x_{\mu}=\sqrt{1+W_{0}(-\exp [-(\bar{\mu}+1)])},
$$

where we take the positive square root and the $W_{0}$ branch, since $p \in \mathbb{R}^{+}$. Since $W_{0}(-1 / e)=-1$, we see that, indeed, $\bar{\mu} \rightarrow 0 \Rightarrow x_{\mu} \rightarrow 0$.

\section{Black body radiation in extra dimensions}

The extremum formula (19) can be applied to the case of black body radiation with extra dimensions, which has been considered in the work of Alnes et al. ${ }^{29,30}$ In Ref. 29 the authors considered radiation consisting of massless particles moving in a $d$-dimensional space, where the particles are described by Bose-Einstein statistics and are in thermal equilibrium at temperature $T$. The energy of a particle with momentum $\mathbf{k}$ is $\omega_{\mathbf{k}}=|\mathbf{k}|=k$, and the internal energy density can be written as ${ }^{29}$

$$
\rho=\frac{\Omega_{d-1}}{(2 \pi)^{d}} \int_{0}^{\infty} \frac{k^{d}}{e^{\beta k}-1} d k .
$$

Using our expression (19), we find that the extremum of such distributions is 


$$
k_{c}=k_{B} T\left[d+W_{j}\left(-d e^{-d}\right)\right], \quad j \in \mathbb{Z},
$$

where we used $k_{B}$ to distinguish the Boltzmann constant from the variable $k$, and $\beta$ above has the same definition as in the present paper.

For black body radiation, the Helmholtz free energy is proportional to the total radiation energy $U$, so that the extremum of the Helmholtz free energy can be found directly from Eq. (19). This also holds for nonextensive thermodynamics, since such proportionality is maintained. ${ }^{31,32}$

\section{FURTHER APPLICATIONS OF THE LAMBERT $W$ FUNCTION TO QUANTUM STATISTICS}

In this section we apply the $W$ function to formulate an expression for temperature $T$ as a function of chemical potential $\mu$ for Maxwell-Boltzmann and Bose-Einstein systems.

\section{A. Maxwell-Boltzmann systems}

For an ideal (perfect) gas, the chemical potential is ${ }^{21}$

$$
\mu=-k T \ln \left[\frac{V}{N}\left(\frac{2 \pi m k T}{\hbar^{2}}\right)^{3 / 2}\right]
$$

which can be written as

$$
-\frac{2}{3} \frac{\mu}{k} \Phi=\Phi T \ln (\Phi T)=e^{\ln (\Phi T)} \ln (\Phi T),
$$

where $\Phi=\left(2 \pi m k / \hbar^{2}\right)(V / N)^{2 / 3}>0$. Applying the $W$ function to both sides of this equation and solving for $T$ one obtains

$$
T=\frac{\hbar^{2}}{2 \pi m k}(N / V)^{2 / 3} \exp \left[W_{j}\left(-\frac{4}{3} \frac{\mu \pi m}{\hbar^{2}}(V / N)^{2 / 3}\right)\right], \quad j \in\{0,1\} .
$$

Or, using the definition of $W(z)$ we can rewrite the solution as

$$
T=-\frac{2}{3} \frac{\mu}{k}\left[W_{j}\left(-\frac{4}{3} \frac{\mu \pi m}{\hbar^{2}}(V / N)^{2 / 3}\right)\right]^{-1},
$$

which is more convenient for computations. We have restricted $j$ to the values corresponding to the purely real branches of the $W$ function, since in the context of classical systems it is assumed that $T, \mu, \Phi \in \mathbb{R}$ (see Figs. 1 and 2).

\section{B. Bose-Einstein systems}

In Sec. II, we have shown that for the Bose-Einstein distribution the density $\rho$ can be expressed as

$$
\rho=\frac{(2 s+1)}{4 \pi^{2}}\left(\frac{2 m}{\hbar^{2}}\right)^{3 / 2} \beta^{-3 / 2} \frac{\sqrt{\pi}}{2} \sum_{k=1}^{\infty} \frac{\left(e^{\beta \mu}\right)^{k}}{k^{3 / 2}} .
$$

Since $\mu<0$, the first term in the sum provides a sufficiently accurate approximation when $\beta=1 / k T \gg 1$. Thus

$$
\rho=A \frac{1}{\beta^{3 / 2}} e^{\beta \mu},
$$

where 


$$
A=\frac{(2 s+1)}{4 \pi^{2}}\left(\frac{2 m}{\hbar^{2}}\right)^{3 / 2} \frac{\sqrt{\pi}}{2}
$$

which can be written as

$$
-\frac{2}{3} \mu \beta e^{-(2 / 3) \mu \beta}=-\frac{2}{3} \mu\left(\frac{\rho}{A}\right)^{-2 / 3} .
$$

Applying the $W$ function to both sides we can find $T$ as a function of $\mu$ :

$$
T(\mu)=-\frac{2}{3}\left(\frac{\mu}{k}\right)\left[W_{j}\left(-\frac{2}{3} \mu\left(\frac{\rho}{A}\right)^{-2 / 3}\right)\right]^{-1}, \quad j \in \mathbb{Z},
$$

where the solution is in terms of the complex branches of the $W$ function when $\mu$ is allowed to be complex valued. The fact that $\mu$ and or $T$ can be complex valued has been used in problems of quantum chromodynamics and Bose-Einstein condensation. ${ }^{33,34}$

\section{CONNECTION TO THE LAMBERT TRANSFORM AND THE POLYLOGARITHM}

As mentioned above, the $W$ function is indirectly connected to the Lambert transform and the polylogarithm function.

\section{A. The Lambert transform}

The Lambert transform is defined in Ref. 35 as

$$
F(x)=\int_{0}^{\infty} \frac{a(t)}{e^{x t}-1} d t, \quad x>0 .
$$

Consider the class of functions $a(t)=A t^{f(r)}$ for some $A \in \mathbb{R}$ and some real-valued, non-negative function $f(r)$. Then the integrand in the transform is essentially the family of functions $h(E=t)$ as defined in Eq. (16), where $\beta=x, \alpha=0$, and $\gamma=-1$. So applying the results of (19), we can express the critical points in $t$ as

$$
t_{c}=\frac{1}{x}\left(f(r)+W\left[-f(r) e^{-f(r)}\right]\right) .
$$

\section{B. The polylogarithm}

The integral representation of the polylogarithm function is given by ${ }^{36}$

$$
\operatorname{Li}_{s+1}(z)=\frac{1}{\Gamma(s)} z \int_{0}^{\infty} \frac{t^{s}}{e^{t}-z} d t, \quad|z|<1, \quad \Re(s) \geq-1 .
$$

Here the integrand can be identified with the expression $g_{s}(\gamma=z, \alpha=0, \beta=1)$. Then we can express the $t$-extremum of the integrand defining the polylog as

$$
t_{c}=s+W_{j}\left(z s e^{-s}\right), \quad j \in Z .
$$

\section{CONCLUSIONS}

In this work, we have considered classical and general statistical distributions and shown that the $W$ function provides an analytical framework for the analysis of energy extrema. Thermodynamic quantities of black body radiation such as the Helmholtz free energy, total radiation energy, entropy, and specific heat are amenable to calculation in this formalism. We have also shown that the $W$ function provides a convenient inversion formula for the temperature in terms of the 
chemical potential for Bose-Einstein and Maxwell-Boltzmann statistics, and including the FermiDirac case will be part of subsequent work. Physical considerations have led to the idea that both chemical potential and temperature can be represented in the complex plane. ${ }^{34,37}$ The multivalued, transcendental $W$ function allows for a natural and unified mathematical representation of physical problems involving such complex variables.

Mathematical functions not only assist the physicist in the analysis of physical phenomena but may also be the unifying thread which stimulates the discovery of new physics. The Riemann $\zeta$ function is a good example that made its advent in quantum physics in the Planck black body radiation formula and has been of fundamental importance in quantum field theory. The renaissance of the $W$ function and its applicability to the formalism of quantum statistics is likely to be another example of such phenomena. Moreover, the $W$ function has an indirect connection to the polylogarithm function, which has shown the underlying logarithmic structure in the quantum statistics of ideal gases. ${ }^{19,20}$ It will not be a surprise to find further diverse applications of the $W$ function in other topics of mathematical and theoretical physics as well as in other disciplines.

\section{ACKNOWLEDGMENTS}

We are grateful to M. H. Lee (U. Georgia, Athens) and S. Joyner (UWO Math. Dept.) and Jesse Tanguay (UWO Physics) for valuable discussions. We also thank the Natural Sciences Engineering Research Council (NSERC), Canada, for providing USRA support for Manuel Gil during the Summer of 2008. We would like to thank the anonymous referee for the helpful comments and review of our manuscript.

${ }^{1}$ J. H. Lambert, Acta Helvetica 3, 128 (1758).

${ }^{2}$ J. H. Lambert, Nouveaux Memoires de l'Academie Royale des Sciences et Belles-Lettres de Berlin (1772).

${ }^{3}$ P. Erdös and A. Rényi, Magy. Tud. Akad. Mat. Kut. Int. Kozl.. 5, 17 (1960).

${ }^{4}$ T. C. Scott, J. F. Babb, A. Dalgarno, and J. D. Morgan III, Chem. Phys. Lett. 203, 175 (1993).

${ }^{5}$ R. B. Mann and T. Ohta, Phys. Rev. D 55, 4723 (1997).

${ }^{6}$ T. C. Scott, R. Mann, and R. E. Martinez, Appl. Algebra Eng. Commun. Comput. 17, 41 (2006).

${ }^{7}$ S. R. Valluri, D. J. Jeffrey, and R. M. Corless, Can. J. Phys. 78, 823 (2000).

${ }^{8}$ J. M. Caillol, J. Phys. A 36, 10431 (2003).

${ }^{9}$ P. Jizba and T. Arimitsu, Physica A 365, 76 (2006).

${ }^{10}$ C. Germani and M. Liguori, Gen. Relativ. Gravit. 41, 191 (2009).

${ }^{11}$ D. Jenn, IEEE Antennas Propag. Mag. 44, 139 (2002).

${ }^{12}$ A. Ortiz-Conde, F. J. G. Sánchez, and M. Guzmán, Solid-State Electron. 47, 2067 (2003).

${ }^{13}$ T. C. Banwell, IEEE Trans. Circ. Syst. 47, 1621 (2000).

${ }_{15}^{14}$ O. Steinvall, Appl. Opt. 48, B1 (2009).

${ }^{15}$ S. R. Cranmer, Am. J. Phys. 72, 1397 (2004).

${ }^{16}$ A. Khare, Fractional Statistics and Quantum Theory (World Scientific, Singapore, 2005).

${ }^{17}$ J. Tanguay, Honors project (Physics 4999 E), University of Western Ontario, April 2009.

${ }^{18} \mathrm{H}$. Bransden and C. J. Joachain, Quantum Mechanics, 2nd ed. (Benjamin Cummings, Menlo Park, 2000).

${ }^{19}$ M. H. Lee, Phys. Rev. E 56, 3909 (1997).

${ }^{20}$ M. H. Lee, J. Math. Phys. 36, 1217 (1995).

${ }^{21}$ F. Reif, Fundamentals of Statistical and Thermal Physics (McGraw-Hill, New York, 1965).

${ }^{22}$ L. Lewin, Polylogarithms and Associated Functions (North Holland, 1981).

${ }^{23}$ A. P. Prudnikov, Y. A. Brychkov, and O. I. Marichev, Elementary Functions, Integrals and Series (Gordon and Breach, New York, 1986), Vol. 1.

${ }^{24}$ R. M. Corless, G. H. Gonnet, D. E. G. Hare, D. J. Jeffrey, and D. Knuth, Adv. Comput. Math. 5, 329 (1996).

${ }^{25}$ R. M. Corless and D. J. Jeffrey, Ontario Research Centre for Computer Algebra (ORCCA) technical report, 2000.

${ }^{26}$ E. M. Purcell and R. V. Pound, Phys. Rev. 81, 279 (1951).

${ }^{27}$ O. Asvany, I. Savi, S. Schlemmer, and D. Gerlich, Chem. Phys. 298, 97 (2004).

${ }^{28}$ H. P. Rojas and L. Villegas-Lelovski, Braz. J. Phys. 30, 410 (2000).

${ }^{29}$ H. Alnes, F. Ravndal, and I. K. Wehus, J. Phys. A: Math. Theor. 40, 14309 (2007).

${ }^{30}$ H. Alnes, F. Ravndal, and I. K. Wehus, Phys. Rev. D 74, 105017 (2006).

${ }^{31}$ D. F. Büyükkiliç and İ. Sökmen, Chaos, Solitons Fractals 13, 749 (2002).

${ }^{32}$ C. Tsallis, J. Stat. Phys. 52, 479 (1988).

${ }^{33}$ M. A. Stephanov, Phys. Rev. D 73, 094508 (2006).

${ }^{34}$ G. E. Cragg and A. K. Kerman, Phys. Lett. A 371, 7 (2007)

${ }^{35}$ A. I. Zayed, Handbook of Function and Generalized Function Transformations (CRC, Boca Raton, 1996).

${ }^{36}$ P. Cartier, Séminaire Bourbaki 2000-2001, 137 (2002).

${ }^{37}$ V. Matveev and R. Shrock, Phys. Rev. E 53, 254 (1996). 